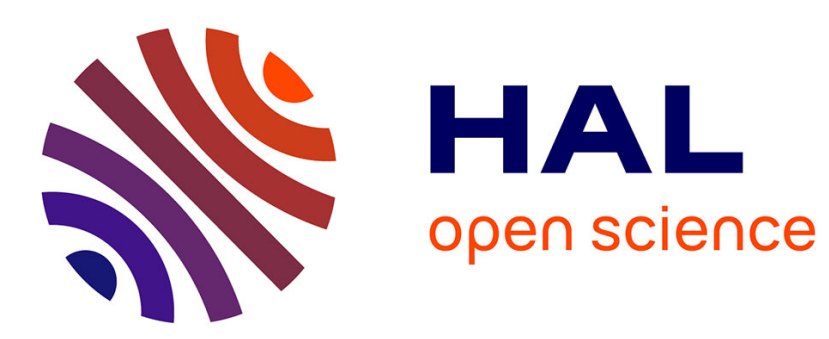

\title{
Agriculture et rapports sociaux de sexe
}

Annie Rieu

\section{To cite this version:}

Annie Rieu. Agriculture et rapports sociaux de sexe: La revolution silencieuse des femmes en agriculture. Cahiers du Genre, 2004, 37, pp.115-130. halshs-00109561

\section{HAL Id: halshs-00109561 https://shs.hal.science/halshs-00109561}

Submitted on 24 Oct 2006

HAL is a multi-disciplinary open access archive for the deposit and dissemination of scientific research documents, whether they are published or not. The documents may come from teaching and research institutions in France or abroad, or from public or private research centers.
L'archive ouverte pluridisciplinaire HAL, est destinée au dépôt et à la diffusion de documents scientifiques de niveau recherche, publiés ou non, émanant des établissements d'enseignement et de recherche français ou étrangers, des laboratoires publics ou privés. 
Agriculture et rapports sociaux de sexe

La « révolution silencieuse « des femmes en agriculture ${ }^{1}$

Annie Rieu

Chargée de recherches CNRS CERTOP/UMR 5044 et équipe Simone/sagesse, Université Toulouse le Mirail

5, Allées Antonio Machado

31058 Toulouse-cedex

0561503653

rieu@univ-tlse2.fr

\section{Introduction :}

Cet article s'appuie sur un corpus de recherches menées depuis 1982 sur le monde agricole, et plus particulièrement sur la situation des femmes dans le cadre de recherches nationales et internationales. La mobilisation de données statistiques permet de suivre les évolutions par rapport aux statuts professionnels., dans un contexte de transformations majeures du monde agricole. Mais les différentes recherches menées sur différents terrains ruraux avec une problématique du «genre » montrent la difficulté pour les femmes de construire une identité professionnelle individuelle, dans un modèle d'agriculture toujours basé sur le paradigme du «métier de couple». En effet, les résistances à l'égalité des sexes perdurent au niveau de la profession agricole, alors que du côté des couples agricoles, on note une évolution dans les

\footnotetext{
${ }^{1}$ Cette formulation est empruntée à Michel Debatisse dans son ouvrage l'agriculture, porte-parole du mouvement des jeunes agriculteurs, devenu en 1964 secrétaire général de la Fédération Nationale des Syndicats d'Exploitants Agricoles (FNSEA).
} 
rapports sociaux de sexe un peu plus rapide. Par leurs exigences en termes de retraite, de protection sociale et de reconnaissance de leur travail, les femmes interpellent fortement leurs conjoints et la profession agricole, pour jeter les bases d'une égalité des sexes.

\section{Changements dans le métier d'agriculteur et professionnalisation des}

\section{femmes :}

Depuis de nombreuses années, le monde agricole a subi de profondes transformations, les paysans sont devenus des agriculteurs, producteurs et «jardiniers de l'espace», les « conjointes » des agricultrices. Au-delà de ces appellations qu'est ce qui a changé dans l'exercice du métier et dans les rapports entre les sexes? Le passage d'une économie dominée par la production de biens à une économie dominée par la production de services (environnement, tourisme, aménagement du territoire) constitue une des transformations majeures de l'évolution de ce secteur. En conséquence les compétences qui devront être mobilisées sont tout autant liées au relationnel qu'à la production stricto sensu et les femmes en sont partie prenante. En parallèle, à la transformation de l'activité agricole en elle-même, de nombreuses exploitations agricoles ont cessé leur activité. Au cours de la période 19881993, le nombre des exploitations de moins de quarante unités de dimension économique(UDE) a diminué fortement (- 38\%), alors que celui des exploitations de plus de cent UDE a progressé de 35\% (Insee,Inra 1998). De façon générale, les grosses exploitations sont gérées par un seul exploitant, souvent l'homme, les épouses exerçant de plus en plus une profession non agricole ${ }^{2}$. De même, les statuts des exploitations se sont diversifiés. Si les entreprises individuelles familiales et conjugales restent dominantes, les formules sociétaires EARL (exploitation agricole à responsabilité limitée) et GAEC (groupement agricole d'exploitation en commun), souvent créées pour des raisons de succession et de fiscalité, 
continuent de progresser (19\% de l'ensemble des exploitations) et les femmes y ont toute leur place $^{3}$ avec des statuts équivalents à ceux des hommes.

Dans les exploitations individuelles, la logique familiale qui organise les rapports de travail et détermine une affectation en fonction du statut familial de chacun tend à perdre du terrain au profit du modèle d'une entreprise rurale dirigée par un chef d'exploitation possesseur d'un diplôme professionnel. L'émergence de ce modèle a permis aux agricultrices de s'engouffrer dans les interstices, et d'affirmer leur insertion professionnelle indépendamment de leur conjoint. Si les femmes sont moins nombreuses à travailler dans l'agriculture que par le passé, celles qui y restent travaillent à temps plein, et revendiquent un statut professionnel à part entière (Lagrave, 1998). Elles se déclarent plus souvent exploitantes qu'aides-familiales, ce qui traduit un changement qualitatif dans l'appréciation qu'elles ont de leur travail et de leur rôle sur les exploitations.

Les résultats du dernier recensement confirment que le nombre des conjointes d'exploitants diminue : 380600 en 2000, contre 713700 en 1988, leur effectif diminuant au rythme annuel moyen de 5,1\% l'an (Agreste 2002). De plus en plus de « conjointes » notamment les jeunes femmes se détournent de l'activité agricole : quatre sur dix travaillent sur l'exploitation en 2000, contre six sur dix en 1998. Parmi les jeunes conjointes, deux groupes sont distingués: d'une part, celles qui se professionnalisent et déclarent une profession principale d'agricultrice avec des statuts divers, et d'autre part celles, deux fois plus nombreuses, qui délaissent l'agriculture y consacrant une faible part, et exerçant une profession hors de l'agriculture. Cette désaffection des femmes pour l'agriculture s'observe aussi dans les déclarations des conjointes ayant une profession principale agricole dont la proportion a diminué par trois entre 1988 et 2000 (Agreste, 2001). Cette diminution touche également les hommes dans une proportion semblable.

3 en 1993 elles représentent 23\% alors qu'elles n'étaient que 14\% en 1988 et leur nombre progresse plus rapidement chez les jeunes générations 
En parallèle, la progression des femmes chefs d'exploitation et co-exploitantes est constante : de 156300 personnes en 1988 elles passent à 178700 en 1990, 186300 en 1995 ; le chiffre est à peu près stable en 2000. Elles représentent 24\% de l'ensemble des exploitants en 2001, contre 18\% en 1990 (Rattin 1997). Cela confirme que les femmes sont bel et bien inscrites dans une logique d'acquisition d'un statut professionnel individualisé) et participent activement au développement de leurs exploitations en prenant en charge de nombreux domaines liés à la diversification. Dans ces contextes mouvants les rapports sociaux de sexe (de genre) se transforment en agriculture, de façon silencieuse. Ils vont dans le sens d'une plus grande autonomie des femmes, même si des résistances demeurent, notamment dans la profession agricole largement masculine et « viriliste ».

Les agriculteurs d'aujourd'hui se voient donc confirmés dans un rôle multi-fonctionnel de producteurs et d'animateurs du monde rural, processus dans lequel les femmes se trouvent de plus en plus souvent en première ligne, y compris dans des registres demeurés longtemps sous leur responsabilité : accueil touristique, productions de qualité, accueil d'enfants en insertion, etc.... Or les compétences requises pour la mise en œuvre de ces nouvelles activités sont pour partie étrangères aux hommes, car il s'agit bien souvent de l'extension du domestique dans la sphère professionnelle (Guillemaut, 2000) et ce domaine relève essentiellement du registre féminin.

Ainsi, l'agriculture devient un métier qui ne fait pas seulement appel aux savoir-faire traditionnels, mais nécessite des qualifications et un certain niveau de compétences dans des registres autres que la production agricole stricto sensu.

\section{L'installation en agriculture : les héritiers dominent toujours}

En 1982, Alice Barthez faisait déjà remarquer que les moments clés du monde agricole sont la succession et le mariage (Barthez, 1982). Si les modes d'installation en agriculture ont subi 
des transformations, la forme dominante reste celle de la transmission préférentielle au garçon qui est socialisé pour reprendre le patrimoine -alors que les filles sont socialisées pour sortir du monde agricole en poursuivant des études. Cette «reproduction sociale» forte est génératrice d'inégalités entre les trajectoires des filles et celle des garçons. Pour ces derniers, le suivi d'une scolarisation à dominante professionnelle se finalise par un retour sur l'exploitation en vue d'une installation. Pour les filles, la scolarité plutôt non agricole les écarte de l'agriculture que certaines d'entre elles réintègrent par le mariage avec un agriculteur, après un passage plus ou moins long par l'emploi salarié. Aujourd'hui, la pénurie de successeurs bouleverse quelque peu les schémas classiques. Ainsi les filles peuvent saisir cette chance si elles le désirent, d'autant plus que la montée du chômage rend de plus en plus difficile leur insertion dans le monde de l'emploi salarié. Par ailleurs, on constate - dans certaines régions - de plus en plus d'installations en agriculture de personnes non issues du milieu agricole. Les chiffres du dernier recensement agricole indiquent que les élèves de l'enseignement agricole issus de familles d'agriculteurs exploitants ne représentent plus que $21 \%$ des effectifs contre $42 \%$ en 1985 . Dans le cadre d'une formation agricole, les filles se retrouvant plus souvent que les garçons dans les filières longues : (36\% contre $13 \%$ des garçons.) et ni le secteur de la production, ni l'installation en agriculture ne représentent les débouchés majoritaires de ces filières.

\section{Les femmes à la recherche de statuts autonomes}

Les premiers résultats du dernier recensement agricole indiquent que plus de 34000 femmes de moins de 40 ans se sont installées sur une exploitation agricole, en 2002 (Agreste 2002). Elles exploitent plutôt des fermes avec des élevages hors sol, des élevages d'ovins et de caprins ou cultivent des vignes. On les retrouve dans les régions du Sud et de l'Ouest et sur des petites structures. Les disparités observées dans les différentes régions s'expliquent principalement, par des facteurs d'ordre démographique et économique. Elles peuvent être 
liées à des politiques volontaristes d'installation ou à une dynamique économique de l'agriculture, qui a un effet d'entraînement sur l'installation des femmes. En 2001, parmi les 582717 chefs d'exploitations, 24\% sont des femmes, soit 139835 personnes, ce nombre reste quasiment stable depuis 2000 (145600) (Rattin 2002). La majorité des femmes chefs d'exploitation sont des exploitantes individuelles, $26 \%$ sont en société, $16 \%$ sont membres d'un groupement agricole d'exploitation en commun (GAEC).

Alors que le nombre de chefs d'exploitation masculin accuse une forte baisse (moins 4,2\% par an), ce maintien des femmes, ne correspond pas nécessairement à une féminisation de l'agriculture en France. En effet, sous l'impulsion de mesures législatives, telle la baisse de l'âge de la retraite à 60 ans, de nombreux hommes ont cédé leur exploitation à leurs épouses, afin que celles-ci puissent continuer d'augmenter pendant quelques années leur capital retraite : en $200032 \%$ des femmes s'installaient par transfert d'exploitation de la part de leur époux (MSA, 2003). Dans le même mouvement, la proportion des coexploitantes progresse fortement, notamment en liaison avec le développement des formes sociétaires. Ce statut ouvre des droits et des responsabilités dont l'étendue dépend de l'implication de l'intéressée dans l'activité agricole. En outre il procure des droits professionnels identiques à ceux du chef d'exploitation. Le nouveau statut de « conjoint collaborateur » instauré par la loi d'orientation agricole de 1999 se substitue à celui de «conjoint participant aux travaux ». il leur permet d'acquérir une retraite. Sans conteste, les différentes lois d'orientation agricole (1980, 1988 et 1999) ont constitué des avancées sur le plan des droits accordés aux femmes. Toutefois, hormis le statut de chef d'exploitation, qui ouvre à tous les droits à égalité avec les hommes, le statut de « de conjoint collaborateur» s'il est intéressant pour les femmes au niveau de la retraite, reste en retrait en ce qui concerne la prestation invalidité, et les conjointes «participant aux travaux » demeurent des «ayants droit» de leur mari. Dans le monde agricole, on est encore loin de droits individualisés en matière de protection sociale (Lagrave 
1987, Barthez 1982). L'accès à ces statuts, qui nécessite l'accord du conjoint, crée une situation de dépendance forte pour les femmes, notamment lorsqu'elles vivent des situations économiques difficiles ou des problèmes au sein du couple. C'est sans doute pour cette raison que l'on compte toujours moins de divorces chez les agriculteurs que dans d'autres catégories socioprofessionnelles.

\section{Une division sexuelle des tâches qui perdure, mais avec moins de rigidité}

\section{qu'autrefois}

Basée sur le modèle idéal de la «complémentarité entre homme et femme » selon la profession agricole, l'exploitation agricole fonctionne en fait sur un rapport familial de production (Barthez, 1982), et non sur un rapport d'emploi classique impliquant contrat de travail et qualification. En fait, ce «métier de couple » idéalisé se fondait sur une hiérarchie entre les sexes: l'homme chef d'exploitation, la femme et les enfants avec des statuts subalternes. En s'appuyant sur le postulat de l'intérêt commun, il nie l'individu en tant que tel, mais surtout les femmes pour lesquelles possibilités de construire une identité professionnelle sont limitées.

Le travail des femmes dans les exploitations est pluriel et largement déterminé par les propriétés de l'exploitation (taille, orientation productive, logique professionnelle) et la trajectoire individuelle (socialisation et formation). Le temps de travail professionnel des agricultrices est évalué en moyenne à $4 \mathrm{~h} 10$ par jour, contre $8 \mathrm{~h} 20$ pour les hommes. L'on sait notamment que dans les exploitations d'élevages, fruitières et de polyculture, les femmes sont plus impliquées dans les activités productives que dans les exploitations céréalières où la quasi-totalité des tâches est mécanisée et peut être réalisée par une seule personne.

Le travail des agricultrices à temps plein reste rare : il concerne $28 \%$ des 168800 conjointes actives en 2000 contre 21\% en 1988 (Agreste 2001). Il est surtout de fait des femmes âgées de 45-55 ans, dégagées des charges familiales liées à la présence des enfants en bas âge. En 
revanche les conjointes de moins de quarante ans travaillent davantage à temps plein en 2000, que par le passé (Agreste 2002). L'interprétation de cette pratique est ambiguë. On aurait pu croire que les femmes qui bénéficient d'un statut autonome travaillent davantage que les conjointes, ce n'est pas le cas. De même la charge d'enfants n'exclut pas un fort investissement professionnel. Effectivement on observe des variations du temps de travail en fonction du statut et des orientations productives : les femmes chefs d'exploitation sont $34,2 \%$ à travailler à temps complet.(Agreste, 2002)

La répartition des tâches sur les exploitations correspond généralement à une division sexuelle classique du travail : aux hommes incombent la responsabilité et la prise en charge des travaux mécanisés de l'extérieur (les femmes intervenant à la demande); aux femmes les travaux de soins aux petits animaux, de garde du troupeau, de récolte des fruits ou de gavage des oies et des canards. La polyvalence qui caractérise le travail des agricultrices est souvent vécue comme déqualifiante: «Tout faire, c'est ne pas avoir de compétences 》 (Rieu 1989). .Autrefois, la division sexuelle du travail était plus marquée et entraînait une forte dépendance des femmes vis-à-vis des conjoints. Elles étaient peu formées ou bien dans les écoles ménagères et, de ce fait, intervenaient peu dans la conduite et la prise de décision des exploitations agricoles. Aujourd'hui, les jeunes femmes sont moins formées dans le domaine agricole que leurs conjoints, elles ont néanmoins un niveau d'études générales supérieur à celui des hommes et équivalent à celui de l'ensemble des femmes françaises (Agreste, 2002). Ce niveau d'études leur permet, quand elles souhaitent s'installer en agriculture, de négocier une place et un statut corrects. Une égalité de statut entre conjoints tout comme la formation technique, et donc les compétences reconnues des femmes a des effets indéniables sur l'organisation du travail. Du coup, le système stable, imposé, rigide de la division sexuelle des tâches traditionnelle, évolue sous la pression des femmes. 


\section{Le travail domestique reste très important et peu partagé}

Tout comme une majorité de femmes en France, les agricultrices doivent gérer la quasitotalité du travail domestique. En 1985-86, ce travail représentait en moyenne 6 h15 par jour, contre $1 \mathrm{~h} 40$ pour les hommes. Des données récentes indiquent qu'en 13 ans, le travail domestique a changé de nature et le temps qui lui est consacré a baissé légèrement (Brousse 2000 : Insee :1999-2000). Les couples d'agriculteurs n'échappent pas à un partage inégal des tâches domestiques, ni à la spécialisation des rôles. Comme dans d'autres milieux sociaux, certaines tâches à « dominante féminine » (cuisine, ménage, linge, etc.) sont du ressort quasi exclusif des femmes qui se trouvent peu secondées, hormis pour les soins et éducation aux enfants, alors que les exploitantes agricoles passent également du temps sur les tâches qui sont à dominante masculine dans d'autres milieux. Elles consacrent $11 \%$ de leur temps à cultiver leur potager (Brousse, 2000). Au total «les hommes qui travaillent à leur compte s'impliquent moins que les salariés dans les tâches domestiques du couple » notamment dans le repassage, le ménage, la vaisselle, la cuisine, les courses. Globalement, chez les couples d'indépendants, l'investissement des hommes dans le travail domestique représente quarante minutes de moins que dans les cas où la femme est salariée. La recherche d'efficacité collective du couple nécessite des arbitrages entre le travail dans l'entreprise familiale et le travail dans l'unité domestique. C'est en fonction de l'investissement dans le travail agricole, que les femmes assument ou non la totalité du travail domestique. En l'absence d'une participation conséquente du conjoint, elles restreignent le travail domestique, mais son importance pénalise tout de même les femmes qui ne peuvent pas s'investir plus intensément dans le travail professionnel. Ainsi, le statut social des agricultrices est marqué du sceau de l'ambiguïté, car il renvoie à une double identité par rapport à la famille et par rapport à l'emploi - salarié ou agricole. 
Sans conteste, l'assignation des femmes au travail domestique se traduit par de nombreuses contraintes, mais n'a pas un caractère irréversible et indépassable. Cependant, les représentations qui s'y associent ont des effets au plan identitaire et se traduisent par les discours ambigus et «justificatifs» des couples. Nous citerons le cas d'un couple d'agriculteurs dont l'homme est chef d'exploitation, adhérent syndical, et dont la femme avec un statut de collaboratrice - ce qui ne l'empêche pas de cumuler plusieurs responsabilités syndicales au niveau départemental et régional. Ils ont quatre enfants.

Même si Madame K. à une charge de travail domestique importante sur le plan matériel et organisationnel, son conjoint a fini par intégrer le travail domestique dans ses réflexions et les problèmes d'articulation des différents temps qu'il pose, pour permettre à sa conjointe d'exercer son mandat syndical. Ainsi, il organise les travaux agricoles en fonction du programme de sa femme et de ses absences. Dans son discours, il relativise l'importance du travail domestique: «les charges domestiques ce n'est pas important, mais ce n'est pas l'esclavage. Ma femme fait le maximum, ... mais, au niveau cuisine on se contente de peu » Sa compagne souligne que son mari est son premier supporter et «qu'il adhère à fond aux idées que la femme a sa place dans la société et qu'il y a des améliorations à faire». Elle salue aussi le fait qu'il n'ait pas hésité «à se mettre un petit peu à l'ombre de la vie publique, ce qui demande une certaine dose de courage dans un pays latin ». Ce témoignage montre que la répartition inégalitaire du travail domestique qui traduit somme toute une «domination masculine »n'exclue pas une ratification des engagements publics de la conjointe. Elle n'empêche pas non plus l'existence d'un relatif équilibre entre le «moi conjugal» et l'épanouissement des identités personnelles (de Singly, 1996). 


\section{Une identité professionnelle en perpétuelle construction}

Nos recherches antérieures ont montré que l'histoire professionnelle des hommes fonctionne en symbiose avec l'histoire de l'exploitation, alors que celle des femmes est ponctuée de ruptures et s'articule d'abord avec les événements familiaux (naissances, décès, etc.) (Rieu 1986 ; 1989). Si le sexe masculin procure de fait une identité professionnelle par le biais du statut préexistant dès la transmission du patrimoine et par le biais du système préférentiel d'héritage à l'aîné des garçons (d'aide familial il devient chef d'exploitation); pour les femmes, l'identité professionnelle est en perpétuelle construction, d'autant qu'elles héritent moins souvent que les hommes de la terre (permanence de la loi salique!). Cette exclusion voulue ou subie des filles de la succession se traduit dans les changements de comportements observés.

Si les difficultés de professionnalisation des agricultrices sont bien connues du point de vue juridique (problème récurrent du statut) et des mentalités (notamment en ce qui concerne la succession), le silence est souvent fait quant au partage du travail domestique. Ce dernier constitue toutefois un obstacle à la professionnalisation des femmes sur les exploitations puisqu'il n'est pas comptabilisé dans le temps de travail, alors que les espaces professionnels et familiaux restent encore largement entremêlés. L'investissement total des femmes dans la production est soumis événements familiaux qui ponctuent le cycle de vie. Plusieurs de nos interlocutrices soulignent qu'elles n'ont pu se professionnaliser qu'après avoir élevé leurs enfants ou qu'elles n'envisageaient pas de choisir entre leur statut professionnel et leur rôle maternel. Au total, il semble que les restrictions à la professionnalisation des épouses d'agriculteurs sont autant de moyens d'affirmer une organisation familiale - conjugale- de la production agricole (Barthez 1982). Si les agricultrices ont amplement souscrit à ce modèle par le passé, elles émettent aujourd'hui davantage de réserves à son égard. En témoigne 
l'augmentation du célibat chez les exploitants pluriactifs dont le pourcentage est passé de $23 \%$ en 1998 à $26 \%$ en 2000.

\section{Compétences sociales et poids de l'environnement professionnel}

Un enjeu majeur de la division sexuelle du travail réside dans la définition des qualifications qui est traditionnellement défavorable aux femmes pour des raisons qui sont liées aux définitions sociales du masculin et du féminin.(Daune-Richard, 1998). Aussi, lorsque nous avions interrogé les agricultrices et leurs conjoints sur les qualités nécessaires à l'exercice du métier, les femmes reprennent des arguments teintés d'essentialisme pour justifier leur place sur l'exploitation ou bien leur non-accès à certaines activités : polyvalence, attention aux autres, capacité de gérer plusieurs tâches à la fois, etc. (Rieu, Cettolo, 2000). C'est aussi l'idée d'une nature différente de celle des hommes qui s'exprime, une définition du féminin qui serait plus proche de la nature, des animaux. On constate que les représentations véhiculées par l'environnement professionnel pèsent largement sur la manière dont les femmes se perçoivent. Ainsi, si les femmes se retrouvent en charge du secrétariat, de la comptabilité ou de certains travaux agricoles c'est «par manque de compétences et de maîtrise suffisante des outils», "parce que c'est "comme ça". Elles parlent aussi d'un manque de force physique et d'une incompétence technique - mécanique ou technologique. Mais l'on sait avec Norbert Elias que «ce n'est pas la faiblesse physique des femmes en soi qui était responsable de la grande différence de pouvoir entre les hommes et les femmes et de l'inégalité sociale qui s'en suivait, mais la structure d'une société dans laquelle la force du muscle et les habiletés de combattants remplissaient la fonction sociale la plus importante » (Elias 2000). Dans les métiers dits «physiques» ou techniques, le manque de force ou l'incompétence supposés des femmes sont encore utilisés pour justifier leur mise à l'écart. Pourtant, elles sont nombreuses à conduire des tracteurs et à utiliser et maîtriser l'ordinateur. 
Cette mise en retrait, de la part des agricultrices, n'est qu'un effet des représentations qu'elles se font d'elles-mêmes, ou le reflet ce que les autres (le monde agricole) a toujours pensé d'elles pour les maintenir dans une position subalterne. Force est de constater que l'on est encore dans le registre d'une construction sociale qui représente les femmes comme techniquement incompétentes (Daune-Richard, 1998).

\section{Les obstacles à l'égalité entre les sexes.}

\section{- La force des représentations sexuées}

Parfaitement conscientes du fait que l'imbrication forte entre le domaine familial et le domaine domestique pèse dans leurs choix professionnels, de nombreuses agricultrices tiennent un discours empreint de contradictions, d'ambivalences, car elles refusent de choisir entre une assignation au domestique et un investissement professionnel total.

Parmi la centaine d'agricultrices rencontrées lors de nos enquêtes, une majorité (des conjointes) se sent en mesure d'assumer le rôle de chef d'exploitation en cas de force majeure, mais fait preuve d'une grande réserve vis-à-vis de cette fonction, dans la mesure où elle est encore largement définie au masculin. C'est justement parce que l'on peut lire une représentation sociale masculinisée du métier d'agriculteur dans leur discours, qu'elles ne souhaitent pas pour autant l'exercer en tant que tel. Si le langage de la nécessité sert souvent de principe explicatif au constat de la division sexuelle de tâches, il n'est pas tout à fait certain que les femmes soient pour autant dans le registre d'une intériorisation des valeurs sociales dominantes. Elles vivent un perpétuel dilemme entre les représentations qu'elles ont d'ellesmêmes, qui sont largement déterminées par l'expérience de vie et par la doxa de sexe (Haicault 1992) et le désir d'être des femmes de la terre bien en phase avec les évolutions de la société. 


\section{Les résistances masculines au changement}

Il suffit d'observer la part des femmes dans les organisations professionnelles de l'agriculture et des syndicats pour constater que la profession reste solidement masculine. Malgré les vœux récurrents exprimés par la profession agricole, peu de place est faite aux femmes et la nomination en 1992 de Christiane Lambert à la tête du Centre National des Jeunes Agriculteurs (CNJA) n'a pas eu d'effet d'entraînement pour d' autres femmes.

Certes, au niveau de leurs exploitations, peu de femmes ont le sentiment de vivre sous le joug de la domination masculine (Bourdieu, 1998). Par ailleurs, les conjoints que nous avons interviewés se proclament dans leur quasi-totalité favorable à l'égalité entre les sexes (Rieu, 1986, 1989 ; Rieu, Cettolo 2000). C'est dire à quel point ils sont en avance par rapport aux instances de décision du monde agricole dont le référentiel reste largement masculin et incapable de penser les femmes en dehors de leur lien conjugal ou familial.

Comme nous l'avons souligné, la structuration de l'agriculture est très masculine, les modalités d'accès particulières contribuent à une forme d'exclusion des femmes ou du moins à leur intégration incomplète. Les femmes sont très peu représentées dans les organisations agricoles, - y compris dans les organisations syndicales qui se veulent progressistes comme la Confédération paysanne- et leur degré de responsabilité y est relativement faible ${ }^{4}$. Par ailleurs, on s'aperçoit que les femmes participent peu aux mouvements de défense portés par les syndicats et sont très peu partie prenantes des actions collectives. Quand elles le sont c'est plutôt au titre d'épouses, de mères. Il reste beaucoup à faire pour parvenir à une représentation satisfaisante des femmes dans ces espaces professionnels, même si ces dernières ne considèrent pas l'investissement des lieux de décisions comme une stratégie d'intégration prioritaire.

\footnotetext{
${ }^{4}$ A la FNSEA, les femmes représentent $40 \%$ des adhérentes et 5,9\% des responsables
} 
Pour conclure

Comme l'attestent les différentes statistiques, la situation des femmes en agriculture se transforme. Si l'on se situe du point de vue des agricultrices rencontrées au cours de nos recherches, cette transformation est particulièrement complexe à analyser. Certaines femmes ont contourné leur marginalisation du domaine agricole en s'inscrivant dans l'emploi salarié (une sur trois chez les jeunes exploitants). L'hypothèse avancée par Delord et Lacombe (88) selon laquelle: " le fait dominant sera dans quelques années le désengagement de plus en plus fort des femmes de l'agriculture du fait de leur autonomisation professionnelle" est en partie confirmée aujourd'hui. Mais il convient de ne pas négliger la ténacité des femmes de la terre à construire un métier au sein même de l'agriculture. Avec la reconversion de certaines fractions du monde agricole, vers les services aux personnes, les agricultrices se trouvent positionnées «au cour des dispositifs de conquête de nouveaux emplois, de la solidarité nationale, de la citoyenneté participative » (Lagrave 1998). Silencieusement, elles ont essayé de survivre, de résister aux sirènes de l'agriculture productiviste qui a largement contribué à leur marginalisation et aujourd'hui elles se retrouvent en pleine lumière, même si les projecteurs ne sont pas braqués sur elles. Même s'il reste encore beaucoup de chemin à parcourir pour parvenir à une égalité entre les sexes, les agricultrices ne sont pas les moins bien loties pour mener à bien l'utopie de l'égalité des sexes.

\section{Références :}

Agreste, (2001) cahiers 3-4, pp 40-41

Agreste (2002) numéro 2

Barthez, A., (1982) Famille, travail et agriculture, Paris, Economica.

Bourdieu, P., (1998), La domination masculine, Paris, Le Seuil.

Brousse, C (2000) La répartition du travail domestique entre conjoints reste très largement spécialisée et inégale, France, Portrait social, Insee : 1999-2000 : 134-151

Debatisse, M., (1963) La Révolution silencieuse, le combat des paysans, Paris, Calman-Lévy. Daune-Richard, A-M., (1998) Femmes et travail, l'enjeu des qualifications, Revue Pour, $\mathrm{n}^{\circ}$ 158 , juin : pp 39-45 
Delord B, Lacombe Ph., (1987), L'agriculture familiale aujourd'hui, renouvellement et activités des ménages, Etudes rurales. $\mathrm{N}^{\circ} 110-111-112$

Delphy, C., (1983) Agriculture et travail domestique, la réponse de la bergère à Engels, Nouvelles questions féministes, $\mathrm{n}^{\circ} 5$ : pp 3-46

Elias, N., (2000) «les transformations de la balance des pouvoirs entre les sexes. Etude sociologique d'un processus à travers l'exemple de l'Etat romain antique », Politix, vol 1,3 $\mathrm{n}^{\circ}$ 51 ,

Guillemaut, F (1998) La place des femmes en milieu rural. Domination masculine et stratégies féminines, DEA de sociologie, Toulouse.

Haicault, M., (1993) Le symbolique dans la complexité des rapports sociaux de sexe. La doxa de sexe et le travail de domination, Congrès AISLF, Les nouveaux mondes et l'Europe, Lyon.

Insee, Inra, (1998) Les campagnes et leurs villes, Portrait social,

Lagrave, R-M., (1987) Celles de la terre, l'invention politique d'un métier, Paris, EHESS,

Lagrave, R-M, (1998) Les agricultrices, les oubliées de la recherche et du féminisme, Lunes, $\mathrm{n}^{\circ} 4$, pp 21-27

Le Quentrec, Y, Rieu, A, (2003) Femmes : engagements publics et vie privée, Paris, Syllepse. Mutualité sociale agricole (2003) le statut de conjoint collaborateur et le rôle de la femme en agriculture en 2001, , Direction des statistiques et des études économiques et financières, Bagnolet.

Rattin, S (1997). «le développement des formules sociétaires revalorise le métier d'agricultrice », Agreste, cahiers n ${ }^{\circ} 21$.

Rattin, S., (2002) L'agriculture au féminin se professionnalise, Agreste, cahiers $n^{\circ} 2$ : pp 1522

Rieu, A, (1986) Agricultrices et professionnalisation, une enquête en Midi-Pyrénées, Revue Géographique des Pyrénées et du Sud-Ouest, tome 57, fasc. 1, pp 89-101, Toulouse.

Rieu, A, (1989) Itinéraires socioprofessionnels et rapports de sexe en agriculture, Recherches féministes, vol 2-2.

Rieu, A, Cettolo, H, (2000) Femmes et hommes dans les petites exploitations agricoles en Midi-Pyrénées, rapport final, contrat SOE2-CT97 3044, juin 195 pages+ annexes, Toulouse.

Scees (1997) Le développement des formules sociétaires revalorise le métier d'agricultrice, les cahiers, numéro 21

Singly (de) François (1996) Le soi, le couple et la famille, Paris, Nathan «Essais et recherches. Sciences sociales ». 
